\title{
A model for evaluation of the electric activity and oxygenation in the erector spinae muscle during isometric loading adapted for spine patients
}

\section{Lars Ekström ( $\square$ lars.ekstrom@orthop.gu.se )}

Goteborgs universitet Institutionen for kliniska vetenskaper

\section{Qiuxia Zhang}

Goteborgs universitet Institutionen for kliniska vetenskaper

Josefin Abrahamson

Goteborgs universitet Institutionen for kliniska vetenskaper

Joel Beck

Goteborgs universitet Institutionen for kliniska vetenskaper

Christer Johansson

Goteborgs universitet Institutionen for kliniska vetenskaper

Olof Westin

Goteborgs universitet Institutionen for kliniska vetenskaper

Carl Todd

Goteborgs universitet Institutionen for kliniska vetenskaper

\section{Adad Baranto}

Goteborgs universitet Institutionen for kliniska vetenskaper

\section{Research article}

Keywords: Spinal stenosis, EMG, NIRS, paraspinal muscle, muscle oxygenation, muscle activity, laminectomy

Posted Date: February 17th, 2020

DOl: https://doi.org/10.21203/rs.2.23688/v1

License: (c) (i) This work is licensed under a Creative Commons Attribution 4.0 International License. Read Full License

Version of Record: A version of this preprint was published at Journal of Orthopaedic Surgery and Research on April 17th, 2020. See the published version at https://doi.org/10.1186/s13018-020-01652-3. 


\section{Abstract}

Background: Simultaneous measurement of electromyography (EMG) and local muscle oxygenation is proposed in an isometric loading model adjusted for patients that have undergone spinal surgery.

Methods: Twelve patients with degenerative lumbar spinal stenosis (DLSS) were included. They were subjected to a test protocol before and after surgery. The protocol consisted of two parts, a dynamic and an isometric lto loading with a time frame of 60 seconds and accompanying rest of 120 seconds. The Ito test was repeated three times. EMG was measured bilaterally at the L4 level and L2 and was recorded using surface electrodes and collected (Biopac Systems Inc). EMG signal was expressed as RMS and median frequency (MF). Muscle tissue oxygen saturation (MrSO 2 ) was monitored using a Near-infrared spectroscopy (NIRS) device ( INVOS ® 5100C Oxymeter). Two NIRS sensors were positioned bilaterally at the L4 level. The intensity of the leg and back pain and perceived exertion before, during and after the test was evaluated with a Visual Analogue Scale (VAS) and Borg RPE-scale, respectively.

Results: All patients were able to perform and complete the test protocol pre-and postoperatively. A consistency of lower median and range values was noted in the sensors of EMG1 (15.3 $\mu \mathrm{V}$, range 4.5-30.7 $\mu \mathrm{V})$ and EMG2 $(13.6 \mu \mathrm{V}$, range $4.0-46.5 \mu \mathrm{V})$ that were positioned lateral to NIRS sensors at L4 compared with EMG3 $(18.9 \mu \mathrm{V}$, range 6.5 $-50.0 \mu \mathrm{V})$ and EMG4 $(20.4 \mu \mathrm{V}$, range 7.5 -49.0 $\mu \mathrm{V})$ at L2. Right and left side of the erector spinae exhibited a similar electrical activity behaviour over time during lto test (60s). Regional MrSO 2 decreased over time during loading and returned to the baseline level during recovery on both left and right side. Both low back and leg pain was significantly reduced postoperatively.

Conclusion: Simultaneous measurement of surface EMG and NIRS seems to be a promising tool for objective assessment of paraspinal muscle function in terms of muscular activity and local muscle oxygenation changes in response to isometric trunk extension in patients that have undergone laminectomy for spinal stenosis.

\section{Introduction}

Qualitative and quantitative research protocols have been proposed and used either in isolation or in combination for functional assessment of the lumbar spine. Subjective assessment has been shown to be limited in determining the muscle functional status, therefore needs other objective methods to be considered. Measuring muscle electric activity with electromyography (EMG), has been shown to be a reliable method and has previously been used in studies for evaluating the functionality of muscles in the lumbar spine (1-8).

The supply of oxygen and nutrients as well as exchange of deposits are key points in maintaining efficient muscle function. Previous studies have shown that in the lumbar spine muscle oxygenation decreased during static contractions using near-infrared spectroscopy (NIRS) that enables non-invasive, real-time monitoring changes in muscle oxygenation. 
The lumbar spine muscles have extensively been investigated but few studies addressed the muscle activity and oxygenation simultaneously, particularly in clinical practice (9-11). To the best of our knowledge, hemodynamic and electrophysiological studies on patients that have previously undergone lumbar spine surgery are limited.

The purpose of this study is to develop and evaluate objective measurements utilizing EMG and NIRS in order to quantify the muscle functional status during isometric trunk extension pre- and postoperatively in a cohort of a limited number of patients operated with laminectomy for symptomatic lumbar spinal stenosis.

\section{Materials And Methods Patient demographics}

Twelve patients were investigated: eight women and four men; mean age 67 (range 52-79) years; mean body height $176(163-184) \mathrm{cm}$; and mean body mass index $26(22-28) \mathrm{kg} / \mathrm{m} 2$. The patients were referred by General practitioners due to confirmed symptomatic DLSS on Magnetic Resonance Imaging (MRI).

\section{Inclusion and exclusion criteria}

Patients with symptom duration of neurogenic intermittent claudication (NIC) 6 month or more that was confirmed by MRI were included in this study. The exclusion criteria were spinal fractures, disc herniation causing sciatica, infection, previous lumbar spinal surgery and degenerative spondylolisthesis greater than grade 1 and cauda equine syndrome.

The present study was approved by the Regional Ethical Review Board.

\section{Surgical procedure}

All patients were operated with open laminectomy either with osteotomy or with resections of the spinal processes.

\section{Test protocol}

Each patient performed identical dynamic trunk flexion-extension movements (dynamic test) and isometric trunk extensions (Ito test) pre- and 3 months post-operatively. Both left and right sides of paraspinal muscle activity and intramuscular oxygenation were simultenously measured before, during and after both the dynamic test and the Ito tests using EMG and NIRS. See Fig. 1 for a schematic flowchart of the test protocol.

\section{Dynamic trunk flexion-extension test}


All patients were instructed to sit in a semi sitting position with $70^{\circ}$ of hip flexion. They were then asked to lift and lower a weight $(7 \mathrm{~kg})$ using a Lojer Pulley 14/20 traction device, from the floor to a specific height (Fig. 2) individualized according to the patient's spinal length in order to obtain a lumbar movement amplitude of $25^{\circ}$ flexion to $5^{\circ}$ extension (12). The test started with a trial for recognition, followed by 2 minutes rest for baseline data. The patients were instructed to keep their arms straight and keep a cyclic pace when performing the flexion-extension movement until fatigue or a maximum of 3 minutes (Fig. 2).

\section{Isometric trunk extension (Ito test)}

The Ito test was performed according to Ito et al. (1996) with the patients in a prone starting position on an examination table (13). A $10 \mathrm{~cm}$ high pad was placed under the lower abdomen to decrease the lumbar lordosis. The arms were kept parallel to the body axis (Fig. 3a). The patients were instructed to lift their upper body off the examination table to an individual adjusted endpoint. The cervical spine was held in a neutral position, looking down and both feet remained on the examination table throughout the entire test until voluntary fatigue or for a maximum of 1 minute (Fig. 3b). The test started with a trial for recognition, followed by 2 minutes rest for baseline data and then three recorded trials with 2 minutes rest in between.

\section{Assessment of paraspinal muscle activity by EMG}

To evaluate lumbar spine muscle activity, all patients were subjected to an EMG examination. Four Bagnoli single differential surface EMG electrodes (DE-2.1, Delsys Inc USA) were placed bilaterally over the paraspinal lumbar muscles at L2-L4 level according to the protocol recommended by SENIAM (www.seniam.org) for evaluation of trunk or lower back muscle EMG activity (Fig. 4). EMG signals were amplified using a Bagnoli-16 unit which has a filter bandwidth of 20 to $450 \mathrm{~Hz}$. Gain was set to $10 \mathrm{k}$. Signals were then transferred through the BNC output connections to a Biopac MP100 (Biopac Systems Inc. USA) using a Universal interface module (UIM100, Biopac Systems Inc. USA). Acqknowledge software by Biopac was used for collecting data and evaluation of RMS. EMG signals were sampled at $1000 \mathrm{~Hz}$.

The root-mean-square (RMS) values of the EMG signal were calculated at time event $=0,5,10,20,30,40$, 50 and 60 seconds. A time window frame of two seconds was used to calculate a mean value at respective time event. EMG RMS values are thus presented as a function of time and merged with oxygen saturation data.

Median frequency (MF) was calculated using a 2 seconds time window in the $5-60 \mathrm{~s}$, of the lto test loading time frame. A regression coefficient, referred as the slope was then calculated for each Ito loading using a linear approximation.

\section{Assessment of paraspinal muscle oxygenation by NIRS}


Localized changes in the paraspinal muscle tissue oxygen saturation $\left(\mathrm{MrSO}_{2}\right)$ were monitored continuously during the dynamic test and the lto test using a NIRS device (INVOS $₫ 5100 \mathrm{C}$ Oxymeter, Somanetics, Troy, Michigan, USA). Two self-adhesive disposable NIRS sensors (Adult SomaSensor ${ }^{\circledR}$ SAFB-SM, Somanetics, Troy, Michigan, USA) were positioned bilaterally over the paraspinal muscles $2 \mathrm{~cm}$ to the left and right of fourth lumbar vertebra (Fig. 4). The INVOS uses two wavelengths between 730 and $810 \mathrm{~nm}$ and two detectors with a center separation from the light source of 30 and $40 \mathrm{~mm}$, respectively. The penetration depth of the INVOS device is roughly 20-25 mm. For each patient, the $\mathrm{MrSO}_{2}$ values were determined every 5 seconds and collected.

\section{Pain evaluation}

The intensity of low back pain (LBP) and leg pain were evaluated with a $10 \mathrm{~cm}$ Visual Analogue Scale (VAS) ranging from 0 (no pain) to 10 (worst imaginable pain), before and after the dynamic test and the Ito test, respectively. The patients rated their perceived exertion with Borg RPE-scale (14), ranging from 6 (very light) to 20 (maximal exertion) after every test phase.

\section{Subcutaneous tissue thickness measurements}

The skin and subcutaneous thickness at the paraspinal muscles at L4-5 was identified and measured using ultrasound (L10-5, Acuson CV70, Siemens Medical Solutions Inc, USA). Blood pressure was measured in the beginning and at the end of the test protocol using a pressure manometer (NAIS, Matsushita, Electronic Works, Japan).

\section{Data analysis}

Data are presented as means and standard deviation (SD) unless otherwise indicated. A paired sample t test was used to compare data between preoperative and postoperative for each time point measured.

For both EMG and NIRS measurements, recorded values at $\mathrm{t}=5 \mathrm{~s}$ are used as a normalizing value and all values recorded at $t=10,20,30,40,50$ or $60 \mathrm{~s}$ are expressed as a percentage of the value at $t=5 \mathrm{~s}$. This procedure is used in the succeeding report of data evaluation unless noted. Statistical significance for all tests was accepted at the $5 \%$ level. The Wilcoxon signed rank test was used for comparison of pre- and post-operative VAS and Pearson's Chi-Square test was used to compare pre- and post-operative RPE. Statistical analysis was conducted with IBM SPSS Statistics for Windows, Version 25.0. Armonk, NY: IBM Corp and Microsoft Excel (2016).

\section{Results}

\section{Test protocol}

All patients were able to perform and complete the test protocol pre- and postoperatively. The time expired between operation and the postoperative test session varied between 84 to 246 days with a median of 108 days.

\section{Assessment of paraspinal muscle activity by EMG}


A consistency of lower median and range values was noted in the sensors of EMG1 (15.3 $\mu \mathrm{V}$, range 4.5$30.7 \mu \mathrm{V})$ and EMG2 $(13.6 \mu \mathrm{V}$, range 4.0 -46.5 $\mu \mathrm{V})$ compared with EMG3 $(18.9 \mu \mathrm{V}$, range 6.5-50.0 $\mu \mathrm{V})$ and EMG4 $(20.4 \mu \mathrm{V}$, range 7.5-49.0 $\mu \mathrm{V})$. Data is presented and shown in Fig. 5. Right and left side of the erector spinae exhibited a similar behaviour and did show a significant difference at time event $t=20,30$, 50 and $60 \mathrm{~s}$ in lto test 2, between pre- and postoperative values. This was not shown in lto test 1 or 3. There were no difference in RMS percentage values of the EMG signal between left and right side muscular activity pre- or postoperatively (Fig. 6). The time dependent behaviour (0-60 s) of the EMG measurements differed slightly between pre- and postoperatively. Preoperatively, the values in Ito test 1 decreased as a function of time, whereas in Ito test 2 they increased and in Ito 3 a more independent behaviour was noted. Postoperatively, a more consistent behaviour was noted where all values did decrease over time in all Ito tests. Median frequency displayed lower values postoperative compared with preoperative in both sensors and in all Ito tests as can be seen in Table 1.

Values presented are mean, error bars: $95 \% \mathrm{Cl}$.

Table 1

Median frequency from EMG3 and EMG4, presented as mean value and standard deviation based on all subjects.

\begin{tabular}{|c|c|c|c|c|c|}
\hline EMG sensor & Ito & Pre & & Post & \\
\hline & & Mean value $(\mathrm{Hz})$ & SD. & Mean value $(\mathrm{Hz})$ & SD \\
\hline \# 3 & Ito 1 & 67.6 & 13.9 & 64.3 & 10.7 \\
\hline & Ito 2 & 70.4 & 13.9 & 65.2 & 9.5 \\
\hline & Ito 3 & 71.0 & 14.1 & 64.0 & 12.7 \\
\hline \# 4 & Ito 1 & 66.9 & 12.4 & 60.8 & 11.2 \\
\hline & Ito 2 & 69.7 & 11.9 & 62.6 & 10.3 \\
\hline & Ito 3 & 68.3 & 12.7 & 62.3 & 12.1 \\
\hline
\end{tabular}

Regional muscle oxygenation as recorded by NIRS showed great similarities between left and right side sensors. Left side sensor (NIRS1) yielded a mean value of $79 \%$ and a range of 53 to $95 \%$. Whereas right side sensor (NIRS2) yielded a mean value of $80 \%$ and a range of 57 to $94 \%$.

The pre- and postoperative $\mathrm{MrSO}_{2}$ trends of the left and right paraspinal muscles over time before, during and after the Ito tests in a representative patient are shown in Fig. 7.

During loading, the mean (average of the three lto tests) relative change in pre- and postoperative $\mathrm{MrSO}_{2}$ for both left and right paraspinal muscles are presented in Fig. 8. Both pre- and postoperative $\mathrm{MrSO}_{2}$ decreased over the Ito test compared to the baseline value at $\mathrm{t}=5 \mathrm{~s}$ and the $\mathrm{MrSO}_{2}$ trends appeared 
similar between sides. Although postoperative $\mathrm{MrSO}_{2}$ value was lower than preoperative values at the end of the Ito test ( $60 \mathrm{~s}$ ) for both sides, no significant differences were observed. During recovery at the cessation of the Ito test, both pre- and postoperative $\mathrm{MrSO}_{2}$ increased compared to the baseline value and it showed a similar trend for both sides as shown in Fig. 8. Although the postoperative $\mathrm{MrSO}_{2}$ value was higher than preoperative value at the end of recovery for both sides, no significant differences were observed.

\section{Pain evaluation}

Pain was reduced postoperatively as implied by the significant difference between pre-and postoperative observations of VAS in the back, both before $(p=0.017)$ and after $(p=0.007)$ Ito tests, as well as of VAS in the leg after the Ito tests $(p=0.043)$ (Table 2). No difference was noted in perceived exertion between pre- and postoperative parameters (Table 2). Before operation, the patients indicated greater pain in the back and leg after performing the Ito tests, compared to before the tests. Though this was not significant. After operation, the patients did not report more pain in the back and leg after performing the Ito tests.

Table 2

VAS and RPE before and after all three Ito tests, pre- versus postoperatively.

\begin{tabular}{|lllll|}
\hline & Ito test & Preop & Post-op & P values \\
\hline VAS back & $\begin{array}{l}\text { Before test 1 } \\
\text { After test 3 }\end{array}$ & $\begin{array}{l}45(0: 70) \\
50(0: 70)\end{array}$ & $\begin{array}{l}0(0: 18) \\
0(0: 18)\end{array}$ & $\begin{array}{l}\mathrm{p}=0.017^{1} \\
\mathrm{p}=0.007^{1}\end{array}$ \\
\hline VAS leg & $\begin{array}{l}\text { Before test 1 } \\
\text { After test 3 }\end{array}$ & $\begin{array}{l}0(0: 42) \\
26(0: 57)\end{array}$ & $\begin{array}{l}0(0: 0) \\
0(0: 0)\end{array}$ & $\begin{array}{l}\mathrm{Ns} \\
\mathrm{p}=0.043^{1}\end{array}$ \\
\hline RPE & After test 1 & $14(13: 17)$ & $13(11: 15)$ & $\mathrm{ns}^{2}$ \\
RPE & After test 2 & $14(13: 17)$ & $13(11: 17)$ & $\mathrm{ns}^{2}$ \\
\hline RPE & After test 3 & $15(13: 17)$ & $13(11: 17)$ & $\mathrm{ns}^{2}$ \\
\hline
\end{tabular}

Values are presented in Median $\left(\mathrm{Q}^{25}: \mathrm{Q}^{75}\right)$. ${ }^{1}$ Wilcoxon signed rank test, pre- vs. post-operative VAS (Visual Analogue Scale). ${ }^{2} \mathrm{Chi}^{2}$-test, pre-vs. post-operative RPE (The Borg rating of perceived exertion).

\section{Thickness of skin and subcutaneous tissue}

Subcutaneous tissue thickness measurements displayed no difference between right side, median $10.5 \mathrm{~mm}$ (range 8.7-20.0 mm) and left side, median $11.2 \mathrm{~mm}$ (range 8.6-20.0 mm).

\section{Discussion}

The main findings in the present study highlights that all patients could perform the experimental protocol both pre- and postoperatively. Furthermore, both EMG and NIRS methods were shown to be able 
to measure the paraspinal muscle activity and changes of oxygenation in response to isometric trunk extensions in patients pre- and postoperatively.

\section{Experimental protocol}

The dynamic trunk flexion-extension test was considered mainly as a warm up for the test subject. The isometric Ito test applied in our experimental protocol was easy to perform, safe and comfortable for both pre- and postoperative patients. Loading time was set to 60 seconds which is well within the endurance time values that Ito et al. reported for female (mean 70.1 seconds) and male (mean 85.1 seconds) with chronic low back pain (CLBP) (13). The majority of the patients were thus believed to be able to perform the test without reaching their endurance limit. However, this finding is not in line with the findings of Demoulin et al. (2008), who showed that the Ito test was less comfortable and more difficult to standardize. The Biering-Sorenser test is often suggested for measuring trunk extensor muscles, particularly in endurance tests when fatigue of the muscles is investigated. The lto test share the same principals, but use greater trunk support and in doing so, become more user friendly in a cohort of LBP patients that might exhibit fear of movement.

\section{General observations}

\section{EMG}

Muscle activity as recorded by EMG and presented as RMS in all 4 measuring sites displayed an activity level within the same range as previous studies, using similar loading mode and protocols $(15,16)$. In general, a strong valid EMG recording was possible from all sensors, but highly dependent on a good reference position (C7) as well as its conductivity. The RMS values recorded varied, dependent on location of sensor, and independent of pre- or postoperative measuring session or lto test. EMG sensor 1 and 2 were placed more lateral and at a lower spinal level than sensor 3 and 4 , thereby recording different anatomical structures. The level of muscle activity is also specific to the loading mode as reported by Tucker $\mathrm{K}$ et al (17). The Ito test used in this study is viewed mainly as an upper body extension movement without any intentional lateral bending or rotation.

\section{NIRS}

Muscle oxygenation responses in the paraspinal muscles showed a rapid decline during the lto test and an increase toward the baseline value during recovery from the lto test (Figure 8). These results indicate an acute imbalance between oxygen supply and oxygen demand in the working muscles during the lto tests (18). Furthermore, increased intramuscular pressure during muscle contractions reduces muscle blood supply and oxygen delivery to the active muscles (19). The rapid increase in muscle oxygenation 
during the immediate post-exercise period counteracts the reduced oxygen supply due to muscle contractions. The muscle oxygenation trends observed in this study were similar for the pre-and postoperative patients across both right and left sides and our results are consistent with previous studies $(9,20)$. There were large variations shown for muscle oxygenation levels among patients because the level of change may reflect a variation in work intensity and may also be influenced by differences in the location of their pain.

\section{Clinical Outcome}

Since the primary objective of the present study was to propose and validate a protocol suitable for evaluation of lumbar muscle functionality during isometric loading, the cohort was limited in terms of numbers of patients. This has an adverse effect on the possibility to draw any statistical significant conclusions from the material. However, trends or associations could be identified and the cohort can provide information for a future power analysis.

\section{Left - right symmetry}

Muscle activity as recorded by EMG and oxygenation are plausible properties that would be able to detect differences in left - right symmetry. No significant EMG difference was shown to occur in observed patients in neither the pre- nor postoperative measurements between left and right side of the lumbar spine in any load block. However, a weak trend was noted in the postoperative group at time event $\mathrm{t}=30$, 40, 50 and 60 in test load block 2 and 3.

\section{Pre - postoperative differences}

In the present study, the time dependent behavior of the muscle activity as recorded by EMG exhibited preand postoperative differences. The postoperative EMG data displayed a more uniform expression since in all Ito tests the values decreased as a function of time. Preoperatively a more inconsistent behaviour was present, in test 1 the values decreased, but in test 2 and 3 the values increased as a function of time. This could speculatively be an expression of fatigue, namely that the RMS may increase over time. A permanent change in spinal muscular morphometry and substance composition has been observed in patients postoperatively. (21-24). MRI and other image generating tools seems to be the dominating means to evaluate such changes, but EMG offers the opportunity to evaluate the functionality of the muscles and particularly time and load dependent properties such as fatigue.

\section{Fatigue}

The moderate isometric load level reached in a Ito test may not be sufficient to initiate a marked fatigue development within 60 seconds and fatigue is therefore not as well detectable as it would with a load 
level in the vicinity of maximal voluntary contraction (MVC.) Changes in MF and RMS values of the EMG signal as a function of time during isometric loading has been previously suggested as being a reflection of muscle fatigue $(4,7)$. However, the observed phenomenon that MF decrease and RMS increase as a function of time appears not to be consistent in the literature. Plausible causes for this could be low load levels, short loading time events or that the investigated muscles exhibit an unknown behaviour, such as pato-physiological behaviour should not be ruled out.

In the present study the cohort where spinal patients diagnosed with spinal stenosis. They are subjected to a fairly low load compared to the MVC, but are still within the ranges that an individual may be subjected to during daily life, i.e $20-40 \%$ of MVC (25). A notable difference between pre- and postoperative MF and RMS inter-relationship was present.

\section{Normalization of data}

In order to evaluate individual properties and compare between individuals a normalizing of data is proposed. The value at 5 seconds is used as a normalizing reference value for all parameters in order to determine a relative expression in percentage as a function of time. The main focus is thereby shifted to the time dependent properties of the parameters.

\section{Limitations}

\section{Sensor placement}

Sensors EMG 1 and EMG 2 were placed lateral to the NIRS sensors, this means that the muscle activity recorded was from a slightly different muscle bulk than the EMG 3 and EMG 4 sensors. A consistently lower RMS value was recorded for EMG 1 and EMG 2 as compared to EMG 3 and EMG 4 throughout the study, indicating a reduction in lateral muscle activity compared with the more centralized positioning of the sensors. This finding has previously been shown by others and is attributed to the anatomy of the erector spinae and multifidus muscle $(26,27)$. The EMG 1 and EMG 2 sensors appeared to be more consistent in picking up electrical environment noise than EMG 3 and EMG 4. Consequently, more of their recordings were rejected in the analysis. Based on these two major findings, it was elected to remove these from the final analysis and propose only use of EMG 3 and EMG 4 sensors in the future studies.

\section{Thickness of the skin and subcutaneous tissue}

The thickness of the skin and subcutaneous tissue ranged from 8.6 to $20 \mathrm{~mm}$ in the present study. We found no correlations between the thickness of the skin and subcutaneous tissue and $\mathrm{MrSO}_{2}$ values.

\section{Technical issues}

The location of EMG sensors were defined and dependent on where the NIRS sensors were placed with reference to the dorsal midline of the subject. Previous test-retest studies of EMG sensors placement yield 
a signal variation expressed as ICC on the order of $0.52-0.91$ which is to be considered when comparisons between different test occasions are made $(5,28)$.

Electrical environmental noise was present mainly on the EMG 1 and EMG 2 sensors which were located lateral to the NIRS sensors and often occurred on slightly high BMI subjects where speculatively the muscle fat ratio is low and more susceptible to electrical interference, therefore creating a poor signal to noise ratio. Quality control of EMG were performed on data derived from the initial resting phase to assure a minimum of electrical noise superimposed on the true muscle signal. Data containing $50 \mathrm{~Hz}$ noise were omitted from the analysis as noted.

\section{Conclusion}

The present study suggests that simultaneous measurements of surface EMG and NIRS is a promising tool for objective assessment of paraspinal muscle function in terms of muscular activity and local muscle oxygenation changes in response to isometric trunk extensions in both pre- and post-operative analysis of patients that have undergone laminectomy for spinal stenosis.

\section{Abbreviations}

EMG: Electromyography; NIRS: Near infrared spectroscopy; DLSS: Degenerative lumbosacral stenosis; NIC: Neurogenic intermittent claudication; RMS: Root mean square; MF: Median frequency; $\mathrm{MrSO}_{2}$ : Muscle tissue oxygen saturation; LBP: Low back pain; VAS: Visual analogue scale; RPE: Rating of Perceived Exertion; MRI: Magnetic resonance imaging; ITO test; Isometric trunk extension test

\section{Declarations}

\section{Acknowledgements}

No

\section{Ethical review committee statement}

The present study was approved by the Regional Ethical Review Board in Gothenburg at the Sahlgrenska Academy, Gothenburg University, Gothenburg, Sweden, (ID number: 753-17).

\section{Authors' contribution}

LE, QZ and JA conceived the study, carried out the tests, analyzed data and wrote the manuscript. CJ analyzed data and critically reviewed the manuscript for intellectual content. CT critically reviewed the manuscript for intellectual content. $A B, J B$ and $O W$ assisted with study design, contributed with writing of 
the manuscript and critically reviewed the manuscript for intellectual content. $A B$ enrolled all patients for the study.

\section{Funding}

This study was financed by grants from the Swedish state under the agreement between the Swedish government and the county councils, the ALF agreement (ID number 238801).

\section{Availability of data and materials}

The datasets used and/or analyzed during the current study are available from the corresponding author on reasonable request.

\section{Ethics approval and consent to participate}

The study was reviewed and approved by the University hospital review board and the ethics committee of our institution. Written informed consent was

obtained from all participating patients.

\section{Consent for publication}

Not applicable

\section{Competing interest}

The authors declare that they have no competing interest.

\section{Authors details}

Institute of Clinical Sciences, Department of Orthopedics, Sahlgrenska Academy, University of Gothenburg and Sahlgrenska University Hospital, Gothenburg, Sweden

* The Carl Todd Clinic, 5 Pickwick Park, Park Lane, Corsham, SN13 0HN, United Kingdom

\section{References}

1. Ahern DK, Follick MJ, Council JR, Laser-Wolston N. Reliability of lumbar paravertebral EMG assessment in chronic low back pain. Arch Phys Med Rehabil. 1986;67(10):762-5.

2. Heydari A, Nargol AV, Jones AP, Humphrey AR, Greenough CG. EMG analysis of lumbar paraspinal muscles as a predictor of the risk of low-back pain. Eur Spine J. 2010;19(7):1145-52. 
3. Humphrey AR, Nargol AV, Jones AP, Ratcliffe AA, Greenough CG. The value of electromyography of the lumbar paraspinal muscles in discriminating between chronic-low-back-pain sufferers and normal subjects. Eur Spine J. 2005;14(2):175-84.

4. Mannion AF, Connolly B, Wood K, Dolan P. The use of surface ENIG power spectral analysis in the evaluation of back muscle function. Development. 1997;34(4):427-39.

5. Mello RGT, Carri IR, da Matta TT, Nadal J, Oliveira LF. Lumbar multifidus and erector spinae electromyograms during back bridge exercise in time and frequency domains. J Back Musculoskelet Rehabil. 2016;29(1):123-33.

6. Oddsson LI, De Luca CJ. Activation imbalances in lumbar spine muscles in the presence of chronic I ow back pain. J Appl Physiol. 2003;94(4):1410-20.

7. Roy SH. The use of electromyography for the identification of fatigue in lower back pain. Motriz, São Paulo. 2003;9(1):15-20.

8. Roy SH, Oddsson LI. Classification of paraspinal muscle impairments by surface electromyography. Phys Ther. 1998;78(8):838-51.

9. Kell RT, Bhambhani Y. Relationship between erector spinae static endurance and muscle oxygenation-blood volume changes in healthy and low back pain subjects. Eur J Appl Physiol. 2006;96(3):241-8.

10. Yamada E, Kusaka T, Arima N, Isobe K, Yamamoto T, Itoh S. Relationship between muscle oxygenation and electromyography activity during sustained isometric contraction. Clin Physiol Funct Imaging. 2008;28(4):216-21.

11. Yoshitake $\mathrm{Y}, \mathrm{Ue} \mathrm{H}$, Miyazaki M, Moritani T. Assessment of lower-back muscle fatigue using electromyography, mechanomyography, and near-infrared spectroscopy. Eur J Appl Physiol. 2001;84(3):174-9.

12. Kankaanpää $\mathrm{M}$, Taimela $\mathrm{S}$, Webber $\mathrm{CL}$, Airaksinen $\mathrm{O}$, Hänninen $\mathrm{O}$. Lumbar paraspinal muscle fatigability in repetitive isoinertial loading: EMG spectral indices, Borg scale and endurance time. Eur J Appl Physiol Occup Physiol. 1997;76(3):236-42.

13. Ito T, Shirado O, Suzuki H, Takahashi M, Kaneda K, Strax TE. Lumbar trunk muscle endurance testing: an inexpensive alternative to a machine for evaluation. Arch Phys Med Rehabil. 1996;77(1):75-9.

14. Borg G. Psychophysical scaling with applications in physical work and the perception of exertion. Scand J Work Environ Health. 1990;16(Suppl 1):55-8.

15. Miura T, Sakuraba K. Properties of force output and spectral EMG in young patients with nonspecific low back pain during isometric trunk extension. J phys Ther Sci. 2014;26(3):323-9.

16. $\mathrm{Ng} \mathrm{J}$, Richardson C. EMG study of erector spinae and multifidus in two isometric back extension exercises. Aust J Physiother. 1994;40(2):115-21.

17. Tucker K, Falla D, Graven-Nielsen T, Farina D. Electromyographic mapping of the erector spinae muscle with varying load and during sustained contraction. J Electromyogr Kinesiol. 2009;19(3):3739. 
18. Chance B, Dait MT, Zhang C, Hamaoka T, Hagerman F. Recovery from exercise-induced desaturation in the quadriceps muscles of elite competitive rowers. Am J Physiol Cell Physiol. 1992;262(3):C766C75.

19. Jensen BR, Jørgensen K, Hargens AR, Nielsen PK, Nicolaisen T. Physiological response to submaximal isometric contractions of the paravertebral muscles. Spine (Phila Pa 1976). 1999;24(22):2332.

20. Albert WJ, Sleivert GG, Neary JP, Bhambhani YN. Monitoring individual erector spinae fatigue responses using electromyography and near infrared spectroscopy. Can J Appl Physiol. 2004;29(4):363-78.

21. Airaksinen O, Herno A, Kaukanen E, Saari T, Sihvonen T, Suomalainen O. Density of lumbar muscles 4 years after decompressive spinal surgery. Eur Spine J. 1996;5(3):193-7.

22. Chen Y-Y, Pao J-L, Liaw C-K, Hsu W-L, Yang R-S. Image changes of paraspinal muscles and clinical correlations in patients with unilateral lumbar spinal stenosis. Eur Spine J. 2014;23(5):999-1006.

23. Fan S, Hu Z, Zhao F, Zhao X, Huang Y, Fang X. Multifidus muscle changes and clinical effects of onelevel posterior lumbar interbody fusion: minimally invasive procedure versus conventional open approach. Eur Spine J. 2010;19(2):316-24.

24. Kramer M, Katzmaier P, Eisele R, Ebert V, Kinzl L, Hartwig E. Surface electromyography-verified muscular damage associated with the open dorsal approach to the lumbar spine. Eur Spine J. 2001;10(5):414-20.

25. Sawai S, Sanematsu H, Kanehisa H, Tsunoda N, Fukunaga T. Evaluation of muscular activity level in daily actions. Japanese Journal of Physical Fitness and Sports Medicine. 2004;53(1):93-105.

26. De Nooij R, Kallenberg L, Hermens HJ. Evaluating the effect of electrode location on surface EMG amplitude of the $\mathrm{m}$. erector spinae p. Iongissimus dorsi. J Electromyogr Kinesiol. 2009;19(4):e257e66.

27. Vink P, Daanen H, Verbout A. Specificity of surface-EMG on the intrinsic lumbar back muscles. Human Movement Science. 1989;8(1):67-78.

28. Brandt M, Andersen LL, Samani A, Jakobsen MD, Madeleine P. Inter-day reliability of surface electromyography recordings of the lumbar part of erector spinae longissimus and trapezius descendens during box lifting. BMC Musculoskelet Disord. 2017;18(1):519.

\section{Figures}




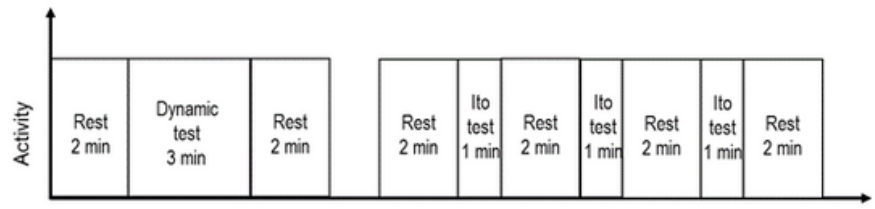

Time (minutes)

\section{Figure 1}

A schematic flowchart of the test protocol. 


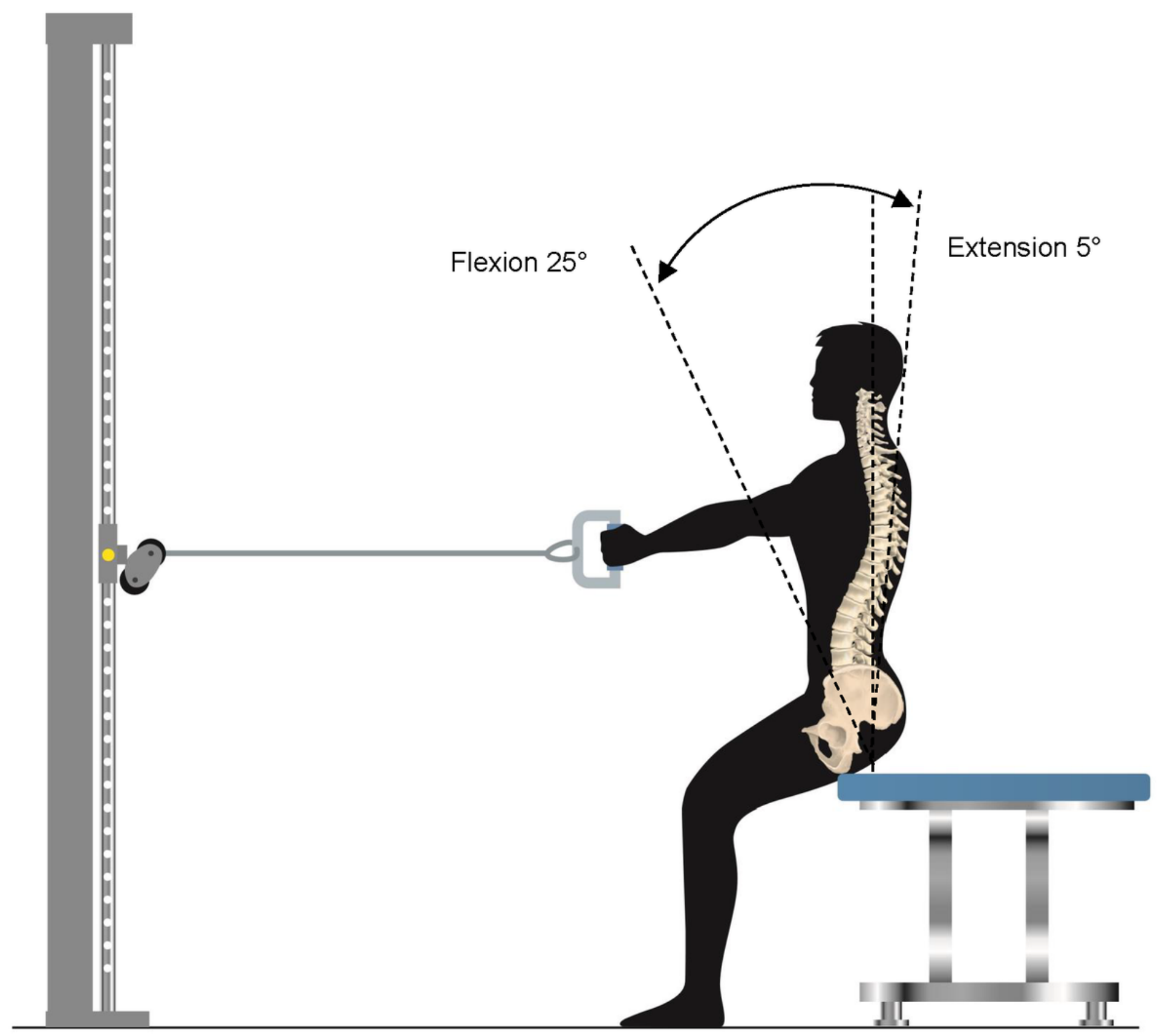

Figure 2

Dynamic trunk flexion-extension test. A full stroke from $25^{\circ}$ flexion to $5^{\circ}$ extension was repeated for 3 minutes at a rate of one per 4 seconds. 

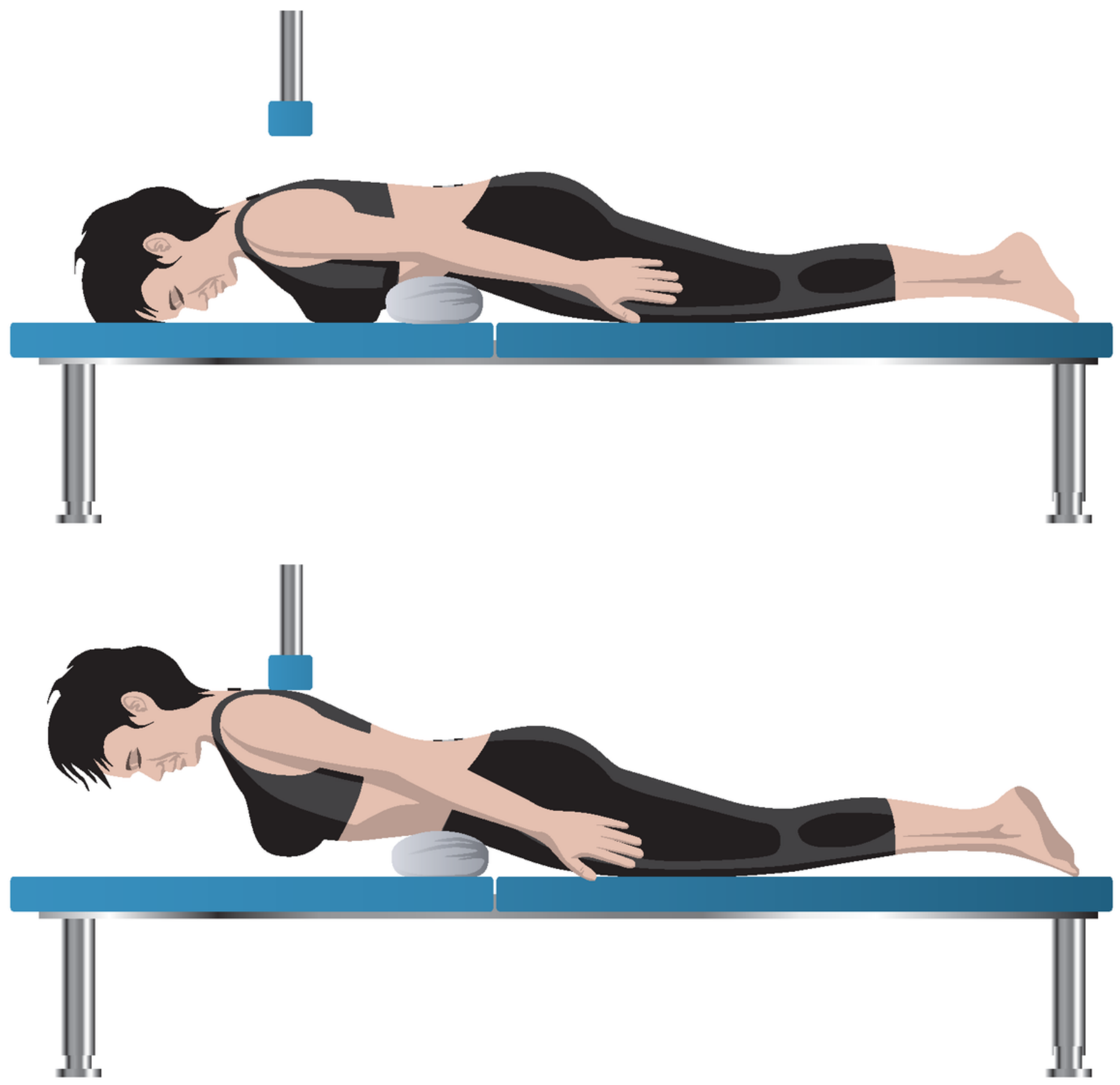

Figure 3

a. Ito test starting position. b. Isometric trunk extension (Ito test), active position held for max 1 min. 


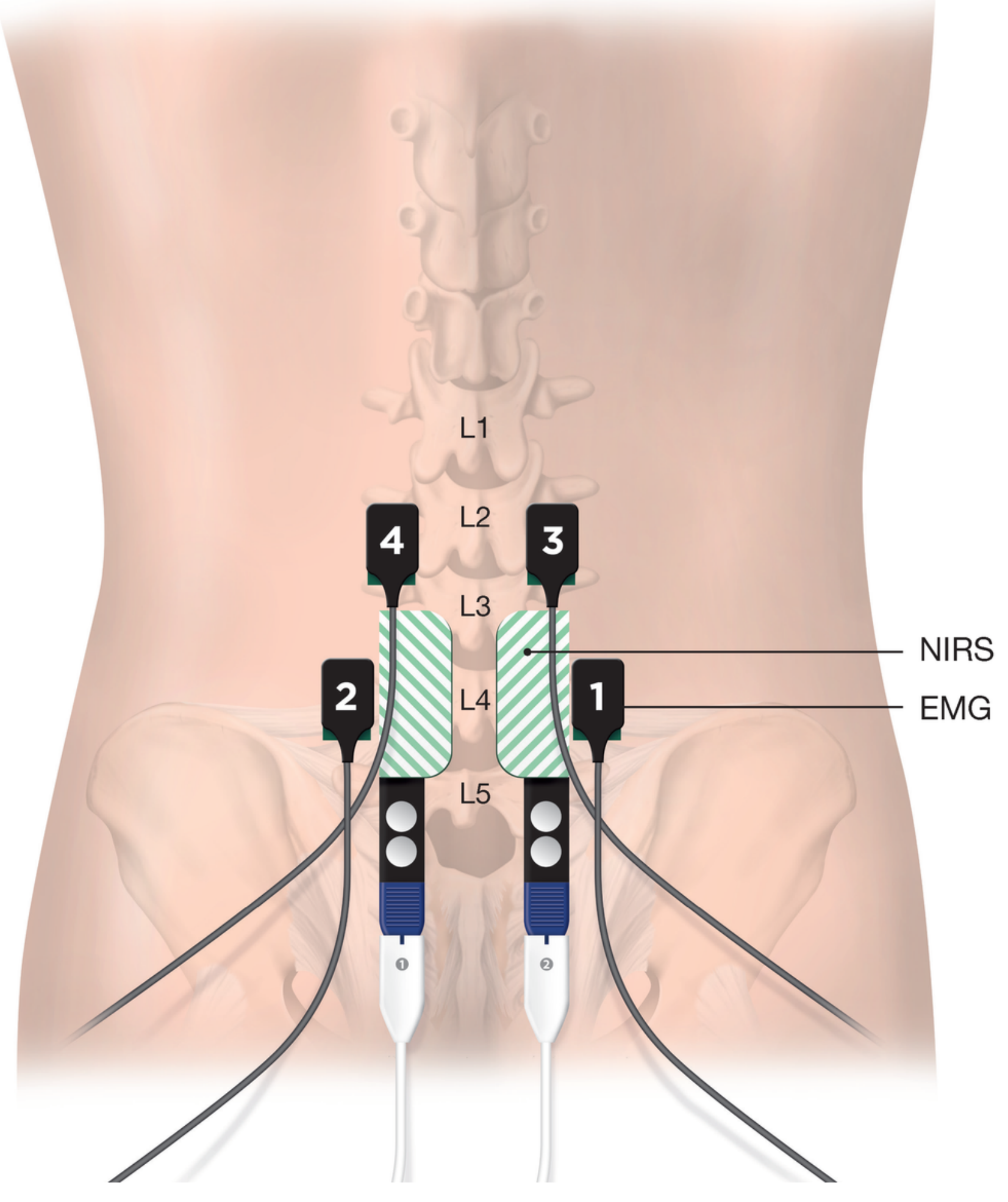

Figure 4

NIRS sensors 1-2 and EMG sensors 1-2 positioned at L4 level. EMG sensors 3-4 positioned at L2. 


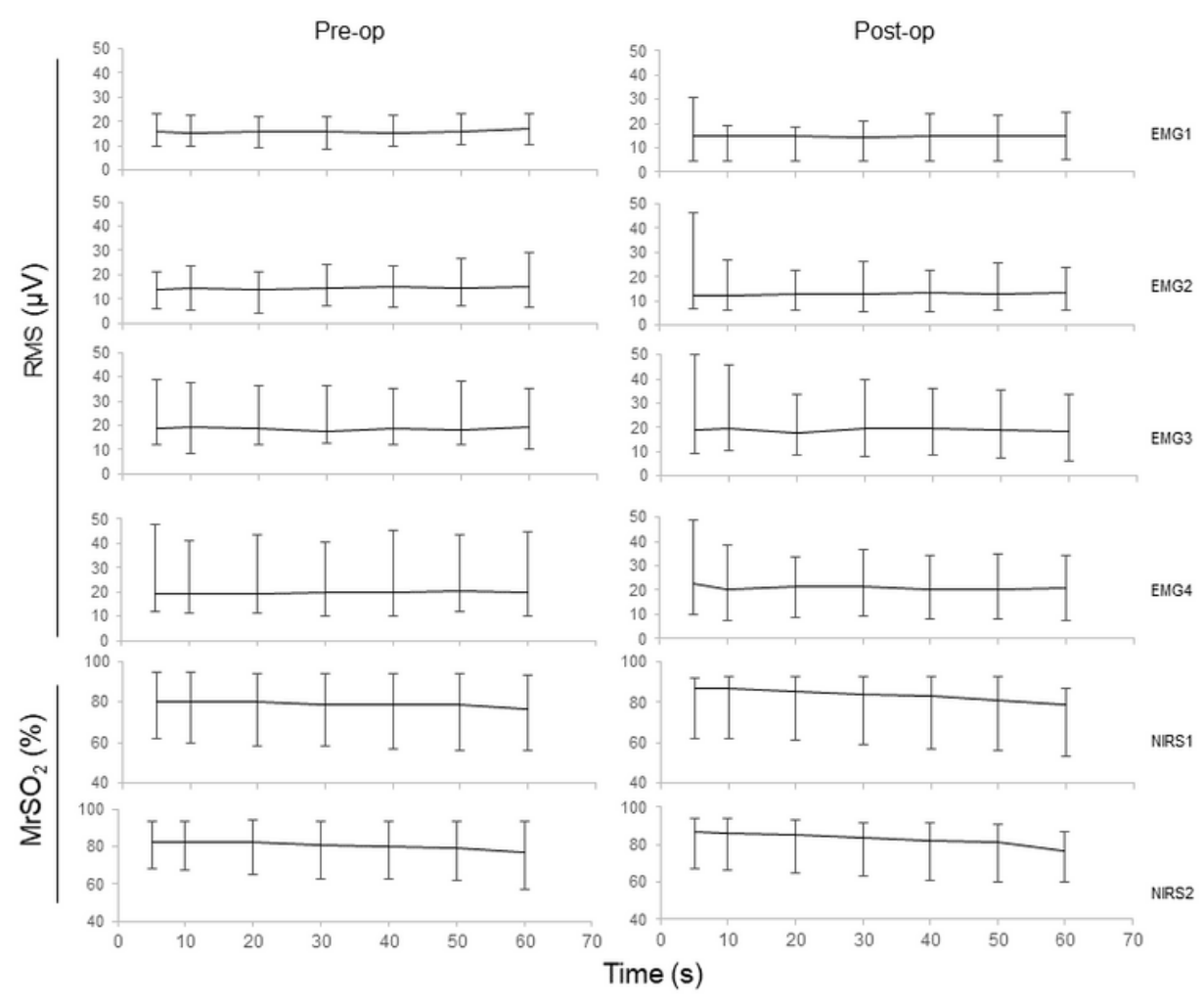

Figure 5

A summary of all collected data as a function of time. EMG $(\mu \mathrm{V})$ and $\mathrm{MrSO} 2(\%)$ values presented as median and range, based on all three lto tests merged for respective sensor.

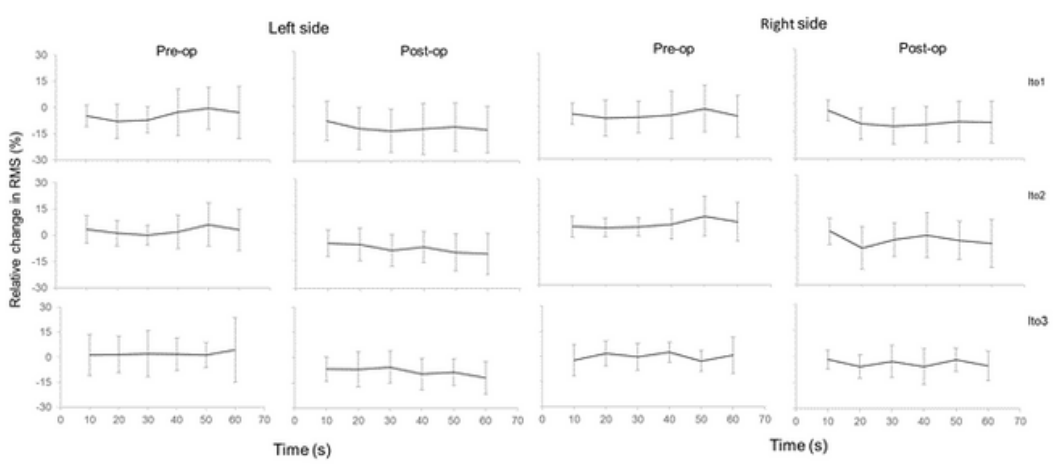


Figure 6

Relative change in RMS expressed as percent over time. Left (EMG4) and right side (EMG3), pre- and post-operatively. All three loading blocks, Ito 1, 2 and 3. Values presented are mean, error bars: $95 \% \mathrm{Cl}$.
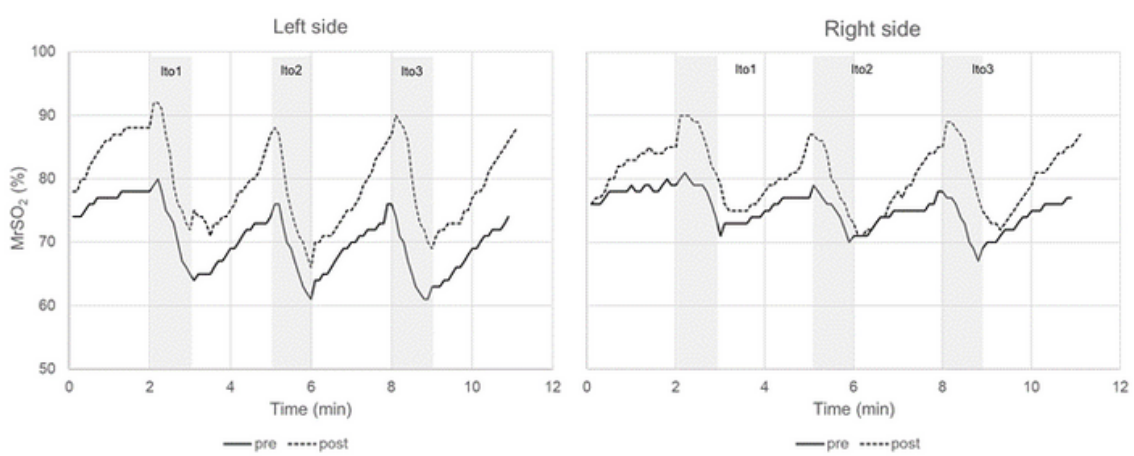

\section{Figure 7}

Original paraspinal muscle regional tissue oxygen saturation (MrSO2) trends of the left and right sides before, during and after the Ito tests in a representative patient. 


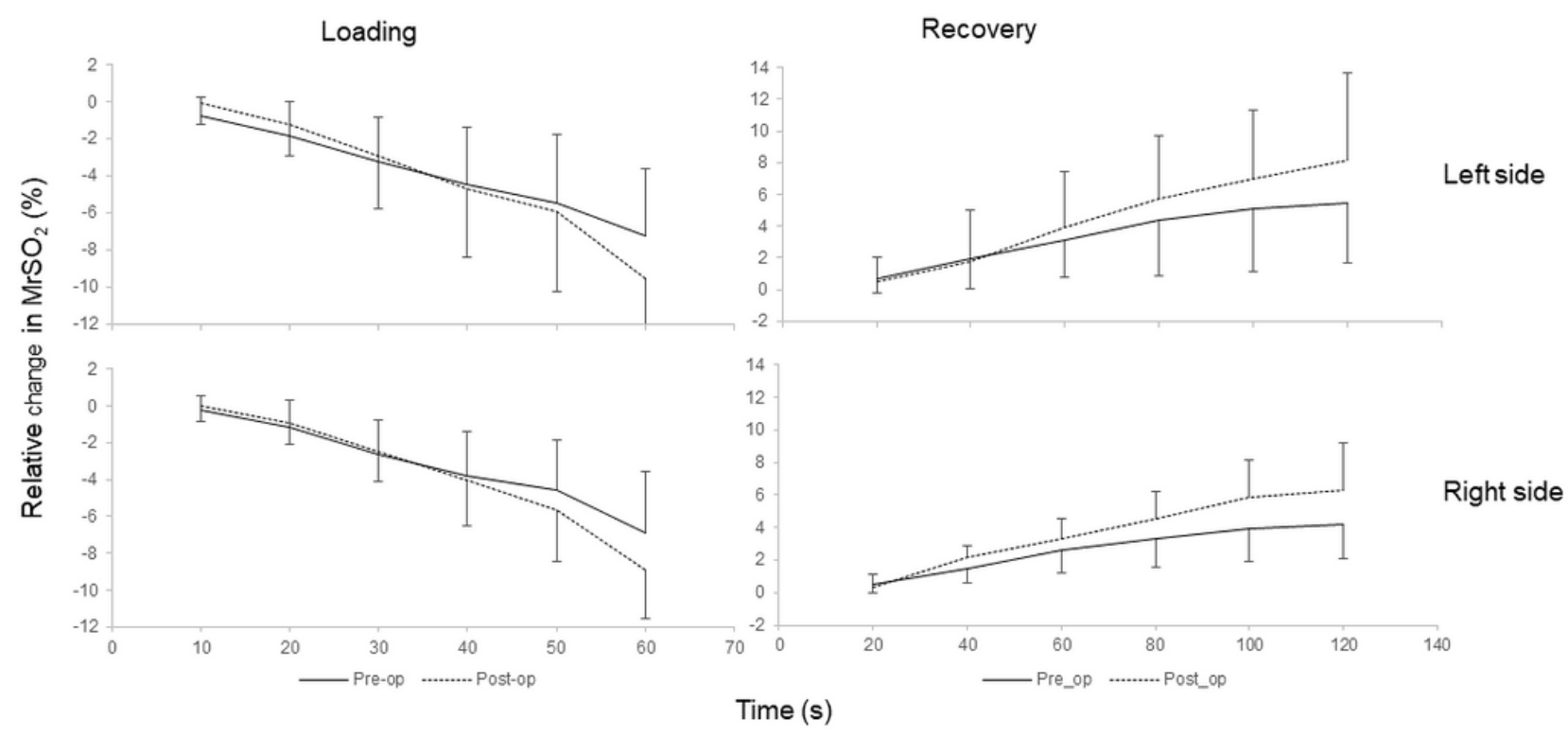

\section{Figure 8}

Relative changes of the paraspinal muscle regional tissue oxygen saturation (MrSO2) during and after the lto test $(n=12)$. The MrSO2 values (mean \pm SD) are expressed as $\%$ of baseline value (value at $5 \mathrm{~s}$ ). 